\title{
Taxonomía y distribución del género Leptoscelis en Costa Rica (Hemiptera: Heteroptera: Coreidae: Leptoscelini)
}

\author{
Taxonomy and distribution of the genus Leptoscelis in Costa Rica (Hemiptera: Heteroptera: \\ Coreidae: Leptoscelini)
}

Harry Brailovsky ${ }^{\bowtie}$ y Ernesto Barrera

Departamento de Zoología, Instituto de Biología, Universidad Nacional Autónoma de México. Apartado postal 70153, 04510 México, D. F., México. \coreidae@ibiologia.unam.mx

\begin{abstract}
Resumen. Se revisa el género Leptoscelis Laporte (Coreidae: Leptoscelini) para Costa Rica y se describe una especie nueva, L. conspicuus. Se discuten sus relaciones con L. quadrisignatus (Distant) y L. tricolor Westwood. Leptoscelis bisbimaculata Breddin es sinonimizada con L. quadrisignatus. Se cita por primera vez L. tricolor para Costa Rica. El trabajo incluye nuevos datos de distribución para el género, ilustraciones de las especies y de los parámeros y una clave para la identificación de las especies costarricenses.
\end{abstract}

Palabras clave: Insecta, Hemiptera, Coreidae, Leptoscelini, Leptoscelis, Costa Rica.

\begin{abstract}
The genus Leptoscelis Laporte (Coreidae: Leptoscelini) from Costa Rica is revised. One new species, L. conspicuus, is described and compared with L. quadrisignatus (Distant) and L. tricolor Westwood. Leptoscelis bisbimaculata Breddin is synonymized with L. quadrisignatus. Leptoscelis tricolor is recorded for the first time from Costa Rica. New distribution records are presented. Habitus illustrations and drawings of parameres are provided. A key to the known Costarican species is presented.
\end{abstract}

Key words: Insecta, Hemiptera, Coreidae, Leptoscelini, Leptoscelis, Costa Rica.

\section{Introducción}

El género Leptoscelis Laporte, 1832 pertenece a la tribu Leptoscelini (Coreidae), y se caracteriza por tener las tibias simples no expandidas, la meso, y metapleura sin manchas discoidales negras, el artejo antenal IV amarillo o rojizo y con la unión basal negra, y el disco pronotal punteado y finamente tuberculado. El género posee 20 especies con una amplia distribución geográfica en el Neotrópico, que va desde Honduras hasta la Argentina y Chile. Para México, Guatemala, El Salvador y las Antillas Mayores y Menores no ha sido registrado aún (Packauskas 2010). Para Costa Rica, solo una especie, L. quadrisignatus (Distant), había sido citada. En esta contribución se describe una nueva especie, se registra por primera vez a L. tricolor Westwood y basándose en el examen de los holotipos L. bisbimaculata Breddin se sinonimiza con L. quadrisignatus.

Acrónimos usados en el texto. BMNH (The Natural History Museum, London); BYU (Brigham Young University, Monte L. Bean, Life Science Museum, Provo, Utah); CASC (California Academy of Sciences, San Francisco);

Recibido: 20 mayo 2011; aceptado: 26 julio 2011
JEEC (Joe E. Eger collection); INBIO (Instituto Nacional de Biodiversidad, Santo Domingo de Heredia, Costa Rica); LACM (Los Angeles County Natural History Museum, California); ROMO (Royal Ontario Museum, Toronto, Ontario, Canadá); SDEM (Senckenberg Deutsches Entomologisches Institut Müncheberg, Alemania); UCDA (University of Davis, California); UMSP (University of Minnesota, Insect Collection, Minnesota); y UNAM (Colección Entomológica, Instituto de Biología, Universidad Nacional Autónoma de México).

\section{Descripción}

Leptoscelis conspicuus n. sp. (Figs. 1-2, 7)

Holotipo macho. Coloración dorsal. Cabeza negra; artejos antenales I a III negros, IV amarillo y con la unión basal negra. Pronoto negro con los márgenes posterolaterales y el margen posterior amarillo. Escutelo amarillo con una mancha central subtriangular cercana al tercio basal, negra. Hemélitro: clavus negro con la comisura claval amarilla; corium negro con el margen apical amarillo; membrana hemelitral pardo oscuro. Abdomen: segmento conexival III negro, IV anaranjado pálido con el tercio anterior negro y los segmentos V a VII anaranjado páli- 
dos; segmentos dorsales anaranjado brillante con manchas negras irregularmente distribuidas. Coloración ventral. Cabeza. Pardo rojizo oscuro, con tercio medio y artejos rostrales I-IV castaño oscuro (ápice del artejo rostral IV, negro). Tórax. Pro-, meso- y metapleura pardo rojizo oscuro; pro-, meso- y metasterno castaño oscuro; lóbulo anterior y posterior del peritremo metatóraxico negros. Patas. Coxa, trocanter, fémur y tíbia pardo rojizo oscuro; tarsos castaño oscuros. Abdomen. Esternitos III a VII y la capsula genital pardo rojizo oscuro; margen pleural III del abdomen pardo rojizo, IV pardo rojizo con el tercio posterior anaranjado y los márgenes V a VII anaranjados. Estructura. Cabeza. Alargada, triangular, apicalmente redondeada, no en declive, finamente tuberculada y sin puntuaciones; depresión postilar con una elevación longitudinal media que separa 2 surcos; tubérculo antenífero inerme; depresión preocelar profunda, circular; tubérculo ocelar elevado; tubérculo postocular redondeado; búcula elevada, corta, posteriormente no rebasando los tubérculos anteníferos y con el tercio anterior angulado; rostro alcanzando el borde posterior del metasterno. Tórax. Pronoto. Trapezoidal, finamente punteado; collar presente; región de los callos sin puntuaciones y con 2 tubérculos anteriores y laterales a la línea media; ángulos frontales redondeados, no expuestos; bordes anterolaterales uniformemente dentados; ángulos humerales ensanchados, lateralmente expandidos y con al ápice subagudo; bordes posterolaterales con la mitad externa recta y dentada y la mitad interna cóncava y lisa; borde posterior recto; metapleura con la espina supracoxal ausente. Patas. Fémures con 2 espinas ventrales largas y cercanas al tercio distal y 2 hileras de espinas pequeñas que corren a lo largo de la superficie ventral; fémur anterior y medio dorsalmente tuberculados; fémur posterior ensanchado, con la superficie dorsal densamente tuberculada y con 3 espinas muy largas y robustas cercanas al tercio basal; tibias anterior y media surcadas, tuberculadas y sin espinas; tibia posterior surcada, con la superficie externa lisa y la interna densamente tuberculada y espinosa. Escutelo. Triangular, apicalmente redondeado. Hemélitro. Macróptero extendiéndose más allá del ápice del abdomen; margen costal del corium emarginado y tuberculado. Abdomen. Ángulo posterior del conexivo inerme y con el borde superior finamente tuberculado. Pigóforo. Borde posteroventral entero, redondeado. Parámero. Figs. 1-2.

Hembra. Color y estructura semejante al holotipo macho. Segmentos dorsales del abdomen VIII y IX pardo oscuro con manchas anaranjadas; segmento conexival VIII pardo oscuro con el tercio anterior anaranjado oscuro y el IX anaranjado oscuro; placas genitales pardo rojizas con el margen externo de la gonocoxa I con tintes anaranjados. Fémures con 2 espinas ventrales largas y cercanas al tercio distal y 2 hileras de espinas pequeñas corriendo a lo largo de la superficie ventral; fémur posterior no ensanchado y con la superficie dorsal sin espinas o tubérculos; tibias surcadas, inermes y prácticamente cilíndricas.

Variación. 1. El rostro alcanza el borde anterior del esternito abdominal III. 2. Margen posterior del pronoto negro. 3. Tibia y tarsos castaño oscuro a anaranjado pálido. 4 . Margen pleural de los esternitos abdominales III y IV negro, el $\mathrm{V}$ negro con el tercio posterior anaranjado, VI y VII anaranjados y VIII y IX negros o amarillo oscuro.

Medidas. Primero del macho después de la hembra. Cabeza. Longitud total: 2.60, 2.57; anchura a través de los ojos: $2.73,2.68$; distancia interocular: 1.47, 1.45; distancia interocelar: $0.62,0.62$; distancia preocular: $1.70,1.60$; longitud de los artejos antenales: I, 3.82, 3.18; II, 3.72, 3.12; III, 2.91, 2.72; IV, 2.82, 2.78. Pronoto. Longitud total: 4.15, 4.08; anchura a través de los ángulos humerales: 7.70, 7.40. Escutelo. Longitud total: 2.70, 2.71; anchura: 2.60, 2.57. Longitud total del cuerpo: $21.55,19.60$.

\section{Resumen taxonómico}

Holotipo macho. Costa Rica. Prov. Puntarenas, Estación Sirena, Parque Nacional Corcovado, 0-100 m, VI-1991, J. C. Saborio (INBIO). Paratipos. Costa Rica. 1 macho, Prov. Puntarenas, Estación Quebrada Bonita, Res. Biol. Carara, 50 m, VI-1990, E. Bello (INBIO); 1 macho, 4 hembras, Prov. Puntarenas, Estación Sirena, Parque Nacional Corcovado, 0-100 m, IV-1989, R. Blanco y G. Fonseca, II-1990, G. Fonseca, X-1990 J. C. Saborio (INBIO, UNAM); 1 hembra, península Osa, Corcovado National Park, Sirena Station, 0-100 m, 08²8' N, 83³5' O, 29-IX-1982, J. Longino (LACM); I macho, península Osa, Rincón, 11-VIII-1966, S. L. Wodd (BYU); 1 hembra, Puntarenas, 1-X-1969, Halffter y Reyes (UNAM); 1 hembra, Prov. Puntarenas, península Osa, 3.5 mi S Rincón, 0842’ N, 83²9’ O, 28-II-12-III-1969, D. C. Rentz (BMNH); 1 hembra, Puntarenas Prov., Corcovado Nat. Park, Sirena Station, 13-29-X-1980, R. W. Mattheus and C. K. Starr (UCDA); 2 hembras, Puntarenas, Quebrada Bonita, Reserva Biológica Carara, $35 \mathrm{~m}, 09^{\circ} 77^{\prime} \mathrm{N}, 84^{\circ} 60^{\prime}$ O, 18-20-V-1990, Holzenthal y Blahnik (UMSP).

Etimología. El nombre específico alude a la conspicua coloración del cuerpo.

Comentarios taxonómicos. Leptoscelis militaris Distant (1901), L. tricolor Westwood (1842) y L. conspicuus n. sp. son especies mayores de $19.50 \mathrm{~mm}$; se relacionan por tener al menos alguna porción del pronoto y del escutelo de color rojo, amarillo o anaranjado y el corium con el margen apical amarillo y nunca provisto de manchas discoidales o de una franja transversal amarilla o roja. Leptoscelis militaris está segregado por que tiene el pronoto, el escutelo y la región pleural del tórax enteramente anaranjado pálido y los esternitos abdominales pardo rojizo. En L. tricolor y L. conspicuus, el pronoto y el escutelo son mayormente negros y los ester- 

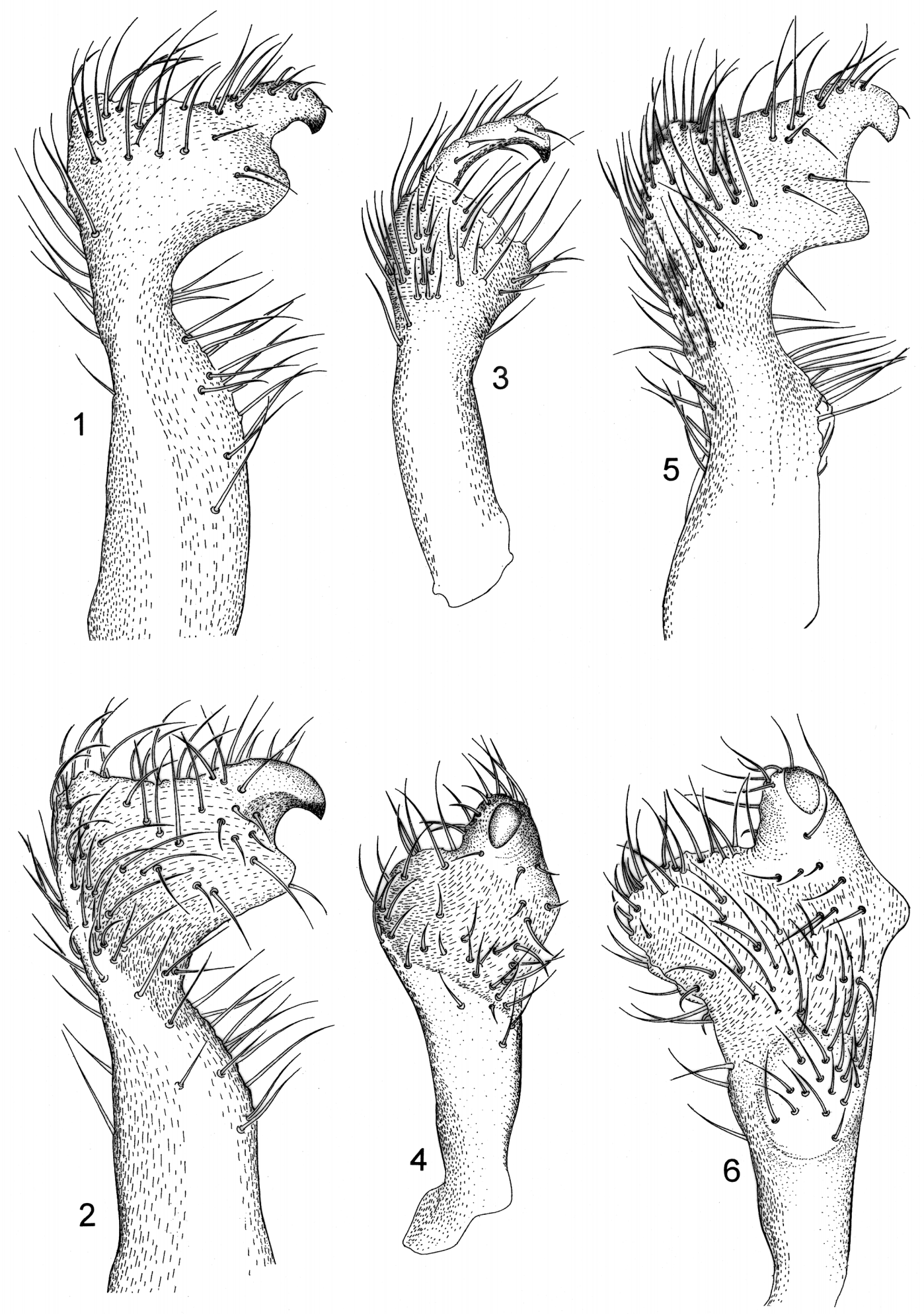

Figuras 1-6. Parámeros en 2 vistas de Leptoscelis spp. 1-2, L. conspicuus n. sp.; 3-4, L. quadrisignatus (Distant); 5-6, L. tricolor Westwood. 

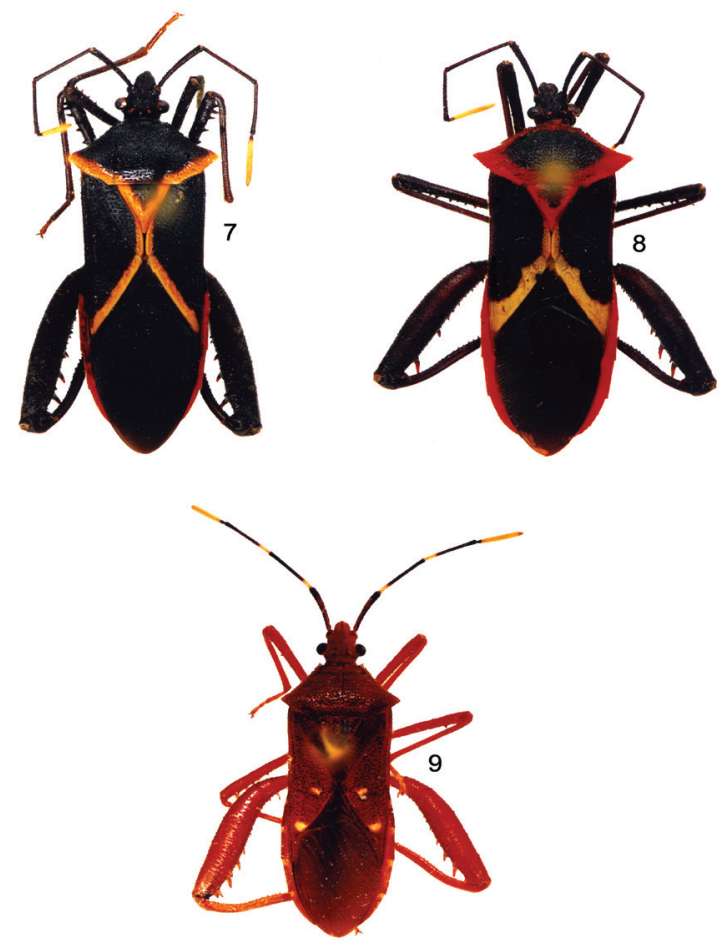

Figuras 7-9. Vista dorsal de Leptoscelis spp. 7, L. conspicuus n. sp.; 8, L. tricolor Westwood; 9, L. quadrisignatus (Distant).

nitos abdominales nunca enteramente pardo rojizo. En $L$. tricolor, la cabeza en vista ventral, el tórax y los esternitos abdominales son totalmente anaranjado rojizo y el pronoto negro con los márgenes anaranjado rojizo, mientras que en L. conspicuus la superficie ventral del cuerpo es pardo rojizo a negro, excepto el margen pleural de los esternitos abdominales IV (sólo el tercio posterior) a VII que son anaranjados; el pronoto es negro, y sólo los márgenes posterolaterales y eventualmente el margen posterior son amarillos (Figs. 7-8). Los parámeros de ambas especies son diferentes; en L. conspicuus, la abertura entre el gancho distal y el lóbulo externo es estrecha, mientras que en L. tricolor es muy amplia (Figs. $1-2,5-6)$.

\section{Leptoscelis quadrisignatus (Distant)}

Laminiceps quadrisignatus Distant 1892: 360.

Leptoscelis bisbimaculata Breddin 1906: 200, syn. nov. (Figs. 3-4, 9)

Coloración dorsal. Anaranjado pardusco con las puntuaciones negras a pardo rojizas. Cabeza. Ocelos rojizos; artejo antenal I anaranjado pardusco con el tercio apical negro, artejos II y III negros con el tercio basal amarillo; el IV amarillo, con la unión basal negra. Pronoto. Ángulos frontales, bordes anterolaterales y ápice de los ángulos humerales amarillos. Hemélitro. Corium con 2 manchas discoidales amarillo cremoso, una cercana al ángulo apical y la otra cerca del endocorium; membrana hemelitral pardo claro. Abdomen. Conexivo anaranjado pardusco con el margen anterior amarillo; segmentos abdominales anaranjado pálido. Coloración ventral. Anaranjado castaño pálido con las puntuaciones negras; lóbulo anterior y posterior del peritremo osteolar negros; artejos rostrales y patas anaranjado pálido; tarsos anaranjado pálido. Estructura. Individuos menores de 18.50 $\mathrm{mm}$. Cabeza. Búcula elevada, corta; el tercio posterior no rebasa los tubérculos anteníferos; el anterior es redondeado y expuesto; el rostro alcanza el tercio medio del esternito abdominal III. Tórax. Pronoto. Ángulos frontales agudos y expuestos; bordes anterolaterales dentados; ángulos humerales ensanchados, lateralmente expandidos y con el ápice subagudo; espina supracoxal de la metapleura presente en los machos. Patas. Fémur anterior y medio escasamente tuberculados; fémur posterior del macho ensanchado, la superficie dorsal densamente tuberculada y con 2 o 3 espinas largas y robustas, cercanas al tercio basal y con la superficie ventral provista de 3 espinas largas, robustas y apicalmente aguzadas. Escutelo. Ápice redondeado.

Hemélitro. Margen costal del corium emarginado y dentado. Abdomen. Borde superior del conexivo prácticamente liso. Pigóforo. Borde posteroventral entero, concavo. Parámero. Figs. 3-4.

Hembra. Coloración y estructura similares a las del macho. Segmentos conexivales VIII y IX, segmentos dorsales del abdomen VIII y IX y placas genitales, anaranjado pálido. Variación cromática. 1. Artejos rostrales anaranjados con tintes rojizos. 2. Ápice de los ángulos humerales negros. 3. Manchas amarillo cremoso del corium marginadas de negro. 4. Tibias anaranjado rojizo oscuro, con la base y el ápice anaranjado pálido.

Medidas. Primero del macho después de la hembra. Cabeza. Longitud total: 2.23, 2.25; anchura a través de los ojos: 2.41, 2.41; distancia interocular: 1.30, 1.32; distancia interocelar: $0.49,0.49$; distancia preocular: 1.44, 1.44; longitud de los artejos antenales: I, 2.72, 2.72; II, 3.28, 2.97; III, 3.03, 2.85; IV, 2.48, 2.25. Pronoto. Longitud total: 3.10, 2.97; anchura a través de los ángulos humerales: 6.01, 5.95. Escutelo. Longitud total: $2.23,2.23$; anchura: $2.17,2.13$. Longitud total del cuerpo: $17.45,17.63$.

Distribución conocida. Esta especie la describe Distant (1892) de Nicaragua (Chontales) y Panamá (Bugaba). Breddin (1906) la cita de Costa Rica (Chiriqui) y Packauskas (2010) de Ecuador y Honduras sin señalar localidad concreta.

Nota. Chiriqui esta situado en Panamá, por lo que la cita de Breddin (1906) es quizás una equivocación en cuanto a la localidad costarricense.

Registros nuevos. Costa Rica. 1 hembra, Prov. Cartago, Turrialba, 610 m, 22-IX-1976, E. S. Ross (CASC); 1 hembra, Prov. Cartago, Turrialba, $2 \mathrm{mi}$ SW of Catie 
Agric. Center, 31-V-1988, B. Harris (LACM); 1 macho, Prov. Heredia, La Selva Biological Station, $3 \mathrm{~km}$ S Puerto Viejo, $10^{\circ} 26^{\prime}$ N, $84^{\circ} 01^{\prime}$ O, 10-IV-1989, H. A. Hespenheide (UNAM); 1 macho, Prov. Heredia, 14 km O Tortuguero, La Suerte Biol. Station, $50 \mathrm{~m}, 10^{\circ} 26^{\prime} 28^{\prime \prime} \mathrm{N}, 83^{\circ} 47^{\prime} 11^{\prime \prime} \mathrm{O}$, 16-19-II-2001, D. C. Darling (ROMO); 6 hembras, Prov. Guanacaste, Estación Cacao, SW side Volcán Cacao, 1 000-1 400 m, III-IV1988, IX-1989, R. Blanco y C. Chávez (INBIO); 1 macho, 2 hembras, Prov. Limón, Cuatro Esquinas, N. P. Tortuguero 0 m, IX-1989, J. Solano (INBIO); 1 macho, Prov. Limón, cerro Tortuguero, Parque Nacional Tortuguero, 100 m, IV-1989, R. Aguilar y J. Solano (UNAM); 1 hembra, Prov. Heredia, finca La Selva Verde, 12 km S Puerto Viejo, 500 ft., 23-26-IX-1986, J. E. Eger (JEEC); 8 machos, 12 hembras, Prov. Heredia, Chilamate, Selva Verde Lodge, 11-13-VIII-1995, J. E. Eger (JEEC); 1 hembra, Prov. Guanacaste, cerro El Hacha, $12 \mathrm{~km}$ SE La Cruz, 300 m, V-1988, M. Espinoza (INBIO); 3 machos, 7 hembras, Prov. Guanacaste, Pitilla, 9 km S Santa Cecilia, 21-III-21-IV-1989 (sin colector) (INBIO); 1 macho, Prov. Heredia, Estación El Ceibo, Parque Nacional Braulio Carrillo, 400-600 m, IX-1989, R. Aguilar y M. Zumbado (INBIO).

Comentarios taxonómicos. El material tipo de L. bisbimaculata Breddin (1906) depositado en SDEM se comparó con el tipo de L. quadrisignatus Distant (1892) depositado en BMNH. Se encontró absoluta concordancia, por lo cual L. bismicamulata se sinonimiza con $L$. quadrisignatus.

Leptoscelis tricolor Westwood

Leptoscelis tricolor Westwood 1842: 17 (Figs. 5-6, 8)

Coloración dorsal. Cabeza. Mayormente negra con el tylus y los juga anaranjado rojizo oscuro y la región del cuello anaranjado rojizo pálido; artejos antenales I-III negros a pardo rojizo y el IV amarillo y con la unión basal negra. Pronoto. Negro y con el margen anterior, los márgenes anterolaterales, los ángulos humerales y los callos anaranjado rojizo (eventualmente el margen posterior también anaranjado rojizo). Escutelo. Anaranjado rojizo y con una mancha negra aproximadamente cuadrada, situada cerca del margen basal. Hemélitro. Clavus negro con el margen de la comisura claval amarillo pálido; corium negro con el margen apical y el ángulo apical amarillo pálido; membrana hemelitral negra. Abdomen. Conexivo anaranjado pálido; segmentos abdominales III a $\mathrm{V}$ anaranjado pálido y con el margen anterior negro, el VI anaranjado pálido con 2 manchas negras anteriores y laterales a la línea media y el VII anaranjado pálido. Coloración ventral. Anaranjado pálido; artejo rostral I anaranjado castaño pálido y los artejos II a IV anaranjado castaño oscuro; coxas anaranjado pálido con tintes rojizos y con el borde externo anaranjado castaño oscuro; trocánteres, fémures, tibias y tarsos pardo rojizos con reflejos anaranjado castaño oscuro. Estructura. Individuos mayores de $19.50 \mathrm{~mm}$. Cabeza. Búcula elevada, corta; el tercio posterior no rebasa los tubérculos anteníferos y el tercio anterior es angulado y expuesto; el rostro alcanza el borde del esternito abdominal III. Tórax. Pronoto. Región de los callos densamente tuberculada; ángulos frontales agudos y expuestos; bordes anterolaterales dentados; ángulos humerales ensanchados, lateralmente expandidos y con el ápice subagudo; espina supracoxal de la metapleura ausente en ambos sexos. Patas. Fémures anterior y medio tuberculados; fémur posterior del macho ensanchado, la superficie dorsal densamente tuberculada y con 2 o 3 espinas largas y robustas cercanas al tercio basal y con la superficie ventral provista de 3 espinas largas, robustas y apicalmente aguzadas. Escutelo. Ápice subagudo. Hemélitro. Margen costal del corium emarginado y dentado. Abdomen. Borde superior del conexivo finamente tuberculado. Pigóforo. Borde posteroventral entero, redondeado. Parámero. Figs. 5-6.

Hembra. Coloración y estructura semejante al macho. Segmentos conexivales VIII y IX, segmentos dorsales del abdomen VIII y IX, y placas genitales anaranjado pálido. Medidas. Primero el macho después la hembra. Cabeza. Longitud total: 2.60, 2.62; anchura a través de los ojos: 2.85, 2.87; distancia interocular: 1.48, 1.55; distancia interocelar: $0.56,0.52$; distancia preocular: $1.69,1.74$; longitud de los artejos antenales: I, 3.65, 3.59; II, 3.78, 3.72; III, 3.16, 3.10; IV, 3.10, 2.97. Pronoto. Longitud total: $4.03,4.15$; anchura a través de los ángulos humerales: 7.85, 7.82. Escutelo. Longitud total: 2.68, 2.72; anchura: 2.72, 2.79. Longitud total del cuerpo: 21.05, 21.82 .

Distribución conocida. Westwood (1842) describe esta especie sin mencionar ninguna localidad o país de origen. Posteriormente fue registrada para Panamá (Bugaba y Chiriqui), Colombia (Bogotá y Nueva Granada), Brasil, Guayana Francesa, Guyana y Surinam (Stál, 1870; Distant, 1881-1892; Blöte, 1936; Packauskas, 2010).

Nuevos registros. Esta especie se cita por primera vez para Costa Rica. Costa Rica. 1 macho, Turrialba, IICA Grounds, 8-XII-1962, C. L. Hogue (LACM); 2 machos, 1 hembra, Prov. Heredia, La Selva Biological Station, $3 \mathrm{~km}$ S Pto. Viejo, $10^{\circ} 26^{\prime}$ N, $84^{\circ} 01^{\prime}$ O, 7-IV-1989, H. A. Hespenheide (UNAM); 1 hembra, Prov. Heredia, Selva Verde Lodge, 11-12-VIII-1995, J. E. Eger (JEEC); 2 machos, 1 hembra, Prov. Cartago, Turrialba, 610 m, 20-IX-1976, E. S. Ross (CASC); 3 machos, Prov. Limón, Estación Hitoy, Cerere, R. Cerere, Res. Biol. Hitoy, 100 m, 12-IV-1992, VI-992, XII-1992, R. Guzmán y G. Carballo (INBIO, UNAM). 
Clave para las especies de Leptoscelis de Costa Rica

1. Artejos antenales II y III negros y con tercio basal amarillo; corium anaranjado pardusco y con 2 manchas discoidales amarillo cremoso; fémures anaranjado pardusco; escutelo anaranjado pardusco............................ quadrisignatus

1'. Artejos antenales II y III negros; corium negro y con margen apical amarillo; fémures negros; escutelo negro y con los márgenes amarillos.

2. Margen anterior y anterolaterales del pronoto negros; tórax y esternitos abdominales pardo rojizo oscuro.

2'. Margen anterior y anterolaterales del pronoto rojos; tórax y esternitos abdominales anaranjado pálido.

L. conspicuus n. sp. .L. tricolor

\section{Agradecimientos}

A los siguientes colegas e instituciones, por haber facilitado en préstamo parte del material aquí estudiado: Mick Webb (BMNH); Richard Baumann (BYU); Norman D. Penny y Vincent Lee (CASC); Jesús Ugalde y Jim Lewis (INBIO); Joe E. Eger (JEEC); Brian Harris (LACM); Brian Hubley (ROMO); Stephan Blank y Angelika Weirauch (SDEM); Steve L. Heydon (UCDA) y Philip J. Clausen (UMSP). A Luis García Prieto (UNAM), por la ayuda computacional brindada.

\section{Literatura citada}

Blöte, H. C. 1936. Catalogue of the Coreidae in the Rijksmuseum van Natuurlijke Histoire. Part III. Coreinae, second part.
Zoologische Mededelingen 19:23-66.

Breddin, G. 1906. Rhynchotographische Beitrage. Wiener Entomologische Zeitung 25:188-200.

Distant, W. L. 1881-1892. Biologia Centrali-Americana. Heteroptera 1. London. 462 p.

Distant, W. L. 1901. LIII.- Rhynchotal Notes.- IX. Heteroptera: Fam. Coreidae. Annals and Magazine of Natural History 7:416-432.

Packauskas, R. 2010. Catalog of the Coreidae, or leaf-footed bugs, of the New World. Fort Hays Studies, fourth series 5:1270.

Stål, C. 1870. Enumeratio Hemipterorum. Kongliga Svenska Vetenskaps-Akademiens Förhandlingar 9:1-232.

Westwood, J. O. 1842. A catalogue of Hemiptera in the collection of the Rev. F. W. Hope, M. A., with short Latin description of the new species. Part II. J. C. Bridgewater, London. 26 p. 